International Journal of Advanced Biological and Biomedical Research

Available online at http:www.ijabbr.com

Volume 8, Issue 2 (2020) pp. 134-145

DOI: 10.33945/SAMI/IJABBR.2020.2.4

Review Article

\title{
Recent Development in Synthesis and Pharmacological Evaluation of Small Molecule to Treat Alzheimer's Diseases: A Review Study
}

\author{
Masome Rahmty \\ Department of Biology, Faculty of Basic Sciences, Islamic Azad University of Central Tehran \\ Branch, Tehran, Iran \\ *Corresponding Author E-mail: MasomeRahmaty0211@gmail.com
}

Received: 05 July 2019, Revised: 25 September 2019, Accepted: 14 October 2019

\begin{abstract}
Alzheimer's disease is a neurological disorder in which the death of brain cells causes memory loss and cognitive decline. It is a neurodegenerative type of dementia, the disease starts mild and gets progressively worse. Like all types of dementia, Alzheimer's is caused by brain cell death. The most common presentation marking Alzheimer's dementia is where symptoms of memory loss are the most prominent, especially in the area of learning and recalling new information.

Alzheimer's disease is not simple to diagnose for which there is no single test. For this reason, the first thing doctors do is to rule out other problems before confirming whether mental signs and symptoms are severe enough to be a kind of dementia or something else. Genetic test is possible in some settings to indicate the likelihood of someone having or developing the disease but this is controversial and not entirely reliable.

There are no disease-modifying drugs available for Alzheimer's disease but some options may reduce its symptoms and help improve quality of life. A different kind of drug, namely memantine, an NMDA receptor antagonist, may also be used, alone or in combination with a cholinesterase inhibitor. This review highlights the several reports that attempt to design and synthesis of some classes of selective Alzheimer's disease inhibitors.
\end{abstract}

Key words: Alzheimer's disease, Dementia, Neurodegenerative disease, $\beta$-amyloid inhibitors

\section{Introduction}

Alzheimer's disease (AD) is a neurological disorder in which the death of brain cells causes memory loss and cognitive decline. AD is the most common type of "dementia" which describes a loss of mental ability related to gradually death of brain cells. It is a neurodegenerative disease, which signals there is the death of progressive brain cell that happens over a course of time (Alzheimer's, 2015). The total brain size shrinks with AD, the tissue has progressively fewer nerve cells and connections. While they cannot be seen or tested in the living brain affected by $\mathrm{AD}$, postmortem/autopsy will always show tiny 
inclusions in the nerve tissue, called plaques and tangles (Aziz et al., 2015). The abnormal protein clumps, are always present with the disease, but there could be another underlying process that actually causes the AD about which scientists are not yet sure (Kumar et al., 2016). Symptoms can be detected at any stage of AD and the progression through the stages of the disease is monitored after an initial diagnosis. Upon the developing symptoms dictate how care is managed. Of course, the very nature of the symptoms can be confusing for both the patients and the people around them, with different levels of severity. For this reason, and because symptoms could signal any of the diagnoses, it is always worthwhile seeing a doctor (Nakanishi et al., 2015).

The most common issues of $\mathrm{AD}$ are when symptoms of memory loss are the most prominent, especially in the area of learning and recalling new information. But the initial presentation might be language problems, with struggling to find the right words. If visuospatial deficits are most prominent, these could include inability to recognize objects and faces, to comprehend separate parts of a scene at once, and a type of difficulty with reading text. Finally, the most prominent deficits in "executive dysfunction" would be to do with reasoning, judgment and problem-solving (Savelieff et al., 2014).

The progression of Alzheimer's can be broken down into three basic stages: Preclinical (no signs or symptoms yet), Mild cognitive impairment and Dementia. The Alzheimer's Association has broken this down further, describing seven stages along a continuum of cognitive decline based on symptom severity from a state of no impairment, through mild and moderate decline, and eventually reaching very severe decline. It is not usually until stage four that a diagnosis is clear-here it is called mild or early-stage Alzheimer's disease, and "a careful medical interview should be able to detect clear-cut symptoms in several areas (Wright et al., 2015). AD is not simple to diagnose with a single test. A gene which called APOE-e4 is related to higher chances of AD affliction among people over the age of 55 years. A lot of clues as to the biological changes in the brain have been uncovered by neuropathology work on brains through examining autopsy. This and other types of research may result in future tests to measure new biomarkers (Wright et al., 2014).

To date, there is no effective cure for AD. The death of brain cells also cannot be compensated. However, therapeutic interventions have been supplied to help AD-afflicted people to live more ably. There are also no disease-modifying drugs available for AD. However emerging options may reduce its symptoms and help improve life quality. There are four drugs in a class called cholinesterase inhibitor approved for symptomatic relief such as Donepezil, Alantamine, Rivastigmine and Tacrine. A different kind of drug, namely memantine as an NMDA receptor antagonist, may also be used alone or in combination with a cholinesterase inhibitor(Kumar et al., 2016). Just as other types of dementia and neurodegenerative disease, a major part of therapy for patients with $\mathrm{AD}$ comes from the support given by healthcare workers to provide dementia quality-of-life care, which becomes more important as independence declines and more support is required.

\section{Alzheimer's disease treatment with small molecule}

\section{$\beta$-amyloid inhibitors}

The $\beta$-amyloid $(\mathrm{A} \beta)$ has been shown to induce neurodegeneration which is determined as the main paradim of $\mathrm{AD}$. It has been indicated that either decrease of $\mathrm{A} \beta$ production or increase of $\mathrm{A} \beta$ clearance is able to enhance AD treatment (Lou et al., 2014a)(Bahramikia et 
al., 2012). The $\beta$-secretase is the enzyme that initiates the generation of amyloid beta. It is an attractive drug target for lowering cerebral levels of APP for the treatment of AD. APP is subjected to degradation via amyloidogenic pathway or the nonamyloidogenic pathway( $\mathrm{Yu}$ et al., 2014b) . Sun and colleges reported that methyl 2-amino-6-(tert-butyl)-4,5,6,7tetrahydrobenzo $[\beta]$ thiophene-3-carboxylate (TBTC), as a selective agonist of retinoid X receptor $\alpha(\operatorname{RXR} \alpha)$ which can effectively activate the heterodimerization of $\operatorname{RXR} \alpha$ with either liver X receptor $\alpha(\mathrm{LXR} \alpha)$, stimulate the expressions of the genes of apoE, ABCA1 and ABCG1, and decrease $\mathrm{A} \beta$ content both in cells and animal models. In addition, administration of TBTC could also reduce the formation of senile plaques (Sun et al., 2015). Herpes simplex virus type 1 (HSV1) infection has been reported to causes the formation of $\mathrm{b}$-amyloid $(\mathrm{Ab})$ and abnormal tau (P-tau). These molecules comprise the main components of the abnormal protein deposits, amyloid plaques and neurofibrillary tangles, respectively, in Alzheimer's disease (AD) brains, and they have been implicated in disease development. The formation of P-tau, but not of Ab, depends on viral DNA replication. Nonetheless, three antiviral agents that inhibit HSV1 DNA replication, including acyclovir (ACV), were found to reduce greatly the level of $\mathrm{Ab}$ as well as $\mathrm{P}$-tau, with the former probably through prevention of viral spread. Previous studies showed that HSV1 DNA is present and is active in the brain of many elderly people, including AD patients, and that in combination with the type 4 allele of the apolipoprotein $\mathrm{E}$ gene, it is likely to play a role in the disease, perhaps via $\mathrm{Ab}$ and Ptau production (Mao et al., 2014a) . With the aim of finding the most suitable antiviral for inhibiting $\mathrm{Ab}$ and P-tau formation as well as HSV1 DNA replication for future use in a clinical trial for treating AD, Wozniak et al. compared the efficacy of ACV with that of another antiviral, BAY 57-1293, which acts by a different mechanism from ACV. In line with previous research, they found that BAY 57-1293 is more efficient than ACV not only in inhibiting HSV1 replication, but also in decreasing Ab and P-tau formation. Also, the cell clusters that are formed during infection are reduced in size much more efficiently by BAY 57-1293 than by ACV. These data suggest that BAY 57-1293 would be a more effective agent than ACV for treating AD (Wozniak et al., 2013). Inhibition of amyloid beta peptide $(\mathrm{A} \beta)$ aggregation is the ultimate goal for slowing $\mathrm{AD}$ progression. In a study, it has been investigated whether the glycoside asiaticoside inhibits $A \beta 1-42$ fibrillation in vitro (Hossain et al., 2015). In addition, Chang et al., hypothesized that conjugation of a metal chelator to destabilize Ab fibrils (fAbs) and a long-chain fatty alcohol to induce neurite outgrowth may generate a novel molecular scaffold that targets both pathologies. The hydroxyalkylquinoline (J2326) was designed and synthesized by joining an 11-carbon alcohol to 5-chloro-8-methoxyquinoline at the 2-position and its antineurodegenerative potentials in vitro and in vivo were characterized. It attenuated fAb formation and disaggregated the existing fAb zinc-dependently as well as zinc-independently. It also triggered extracellular signal-regulated kinase-dependent neurite outgrowth and increased synaptic activity in neuronal cells. In fAb-driven neurodegeneration in vitro, J2326 reversed neurite collapse and neurotoxicity. These roles of J2326 were also demonstrated in vivo and were pivotal to the observed improvement in memory of mice with hippocampal fAb lesions (Figure 1). These results show that the effectiveness of J2326 on fAb-driven neurodegeneration is ascribed to its novel scaffold. This might give clues to evolving attractive therapy for future clinical trials (Chang et al., 2015).

Inhibition of the interaction between $\mathrm{A} \beta$ and a neuronal cell surface receptor served as an important strategy. For instance, LilrB2, has been mentioned as a potential target for 
treating $\mathrm{AD}$, because interaction between $\mathrm{A} \beta$ and LilrB2, triggers a pathway to synaptic loss. Therefore the LilrB2 binding moieties of $\mathrm{A} \beta$ with identified structure, be ${ }^{16} \mathrm{KLVFFA}^{21}$, has been used to inhibit this interaction and its efficacy has been studied in vivo and in vitro. This study showed that these inhibitors are potential therapeutic agents against AD (Cao et al., 2018).
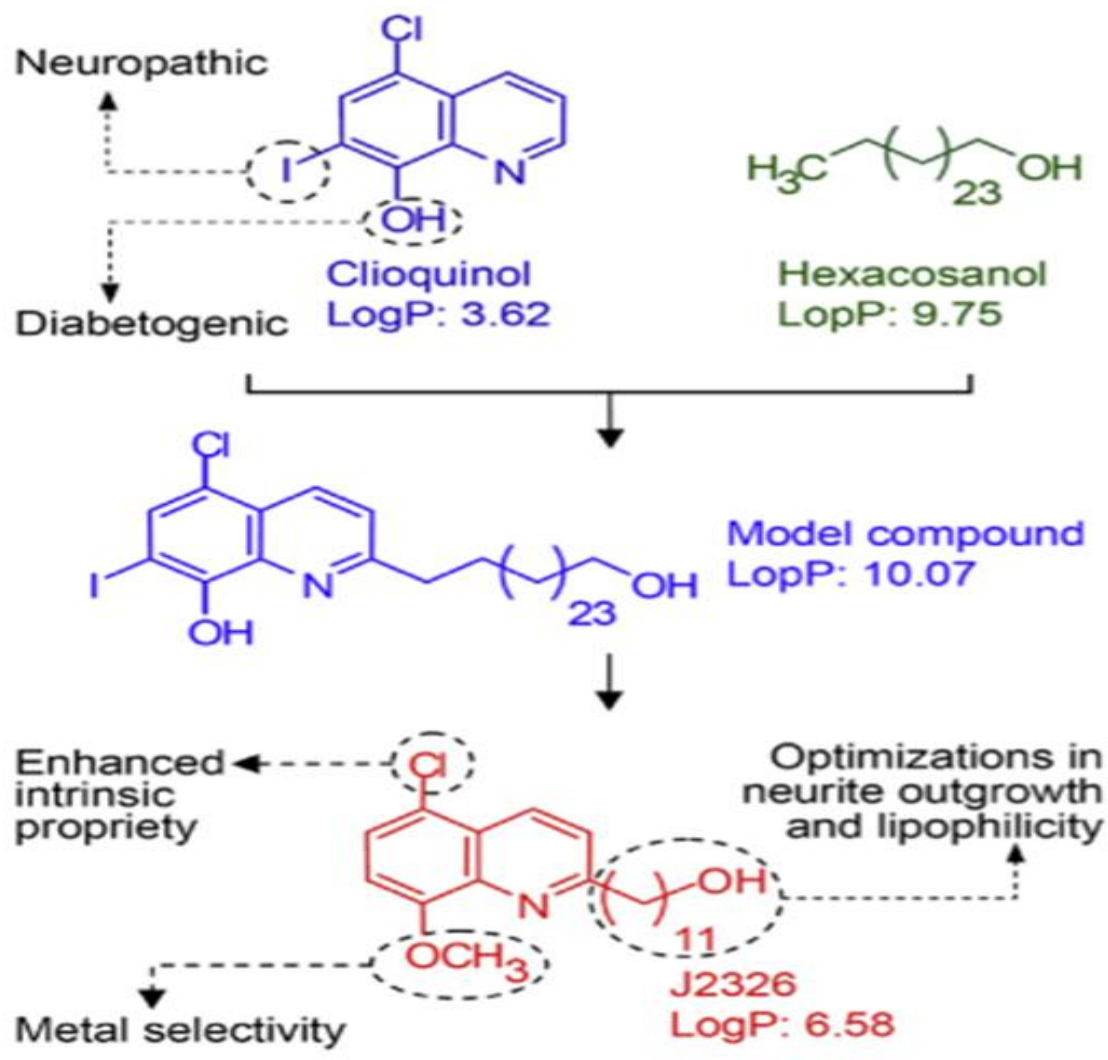

\section{Figure 1.}

Multiple factors, including amyloid-b (Ab), metals, and reactive oxygen species (ROS), are involved in the development of Alzheimer's disease (AD). Metal ions can interact with $\mathrm{Ab}$ species generating toxic oligomers and ROS in vitro (Yu et al., 2014a). However, the involvement of metal-Ab complexes in $\mathrm{AD}$ pathology in vivo remains unclear. To solve this uncertainty, Beck et al., have developed a chemical tool (L2-b) that specifically targets metal-Ab complexes and modulates their reactivity (i.e. metal-Ab aggregation, toxic oligomer formation, and ROS production). Through the studies presented herein, we demonstrate that L2-b is able to specifically interact with metal-Ab complexes over metalfree $\mathrm{Ab}$ analogues, redirect metal-Ab aggregation into off-pathway, nontoxic less structured $\mathrm{Ab}$ aggregates, and diminish metal-Ab-induced ROS production, with overall mitigating metal-Ab-triggered toxicity which is confirmed by multidisciplinary approaches. Most importantly, upon treatment of 5XFAD AD mice with L2-b, (i) metal-Ab complexes are targeted and modulated in the brain; (ii) amyloid pathology is reduced; and (iii) cognition deficits are significantly improved. To the best of our knowledge, by employing an in vivo chemical tool specifically prepared for investigating metal-Ab complexes, Beck et al., 
presents for the first time experimental evidence supporting that metal-Ab complexes are related directly to AD pathogenesis (Beck et al., 2015).

An important factor that may affect the severity and time of onset of Alzheimer's disease (AD) is chronic stress. Epidemiological studies report that chronically stressed individuals are at an increased risk for developing AD(Alkadhi and Tran, 2015). In another study, Alkadhi and associates studied that whether chronic psychosocial stress could hasten the appearance of $\mathrm{AD}$ symptoms including changes in basal levels of cognition-related signaling molecules in subjects who are at risk for the disease. They investigated the effect of chronic psychosocial stress on basal levels of memory-related signaling molecules in area CA1 of subclinical rat model of AD. Western blot analysis of basal protein levels of important signaling molecules in hippocampal area CA1 showed no significant difference between the subclinical AD rat model and control rat. Following six weeks of psychosocial stress, molecular analysis showed that subclinical animals subjected to stress have significantly reduced basal levels of $p$-CaMKII and decreased $p$-CaMKII/t-CaMKII ratio as well as decreased basal levels of p-CREB, total CREB, and BDNF. The results suggest that these changes in basal levels of signaling molecules may be responsible for impaired learning, memory, and LTP in this rat model, which support the proposition that chronic stress may accelerate the emergence of AD in susceptible individuals (Alkadhi and Tran, 2015).

Based on the 'multi-target-directed ligands' (MTDLs) strategy, Mao and his research group designed and synthesised a series of new compounds against AD by combining the pharmacophores of resveratrol and clioquinol. Among these compounds, (E)-5-(4hydroxystyryl) quinoline-8-ol showed the most potent ability to inhibit selfinduced $\mathrm{A} \beta$ aggregation (IC50 $=8.50 \mu \mathrm{M}$ ) and copper(II)-induced $\mathrm{A} \beta$ aggregation and to disassemble the well-structured $\mathrm{A} \beta$ fibrils generated by self- and copper(II)-induced $\mathrm{A} \beta$ aggregation (Figure 2) (Mao et al., 2014b). Previously it has been shown that molecular deletion of plasminogen activator inhibitor 1 PAI- 1 significantly reduced brain $\mathrm{A} \beta$ load in animal model (Angelucci et al., 2019). In this regard, oral administration of TM5275, a small molecule inhibitor of PAI-1, has been indicated to reduce $\mathrm{A} \beta$ load in the hippocampus and consequently improve memory function in animal model (Akhter et al., 2018).

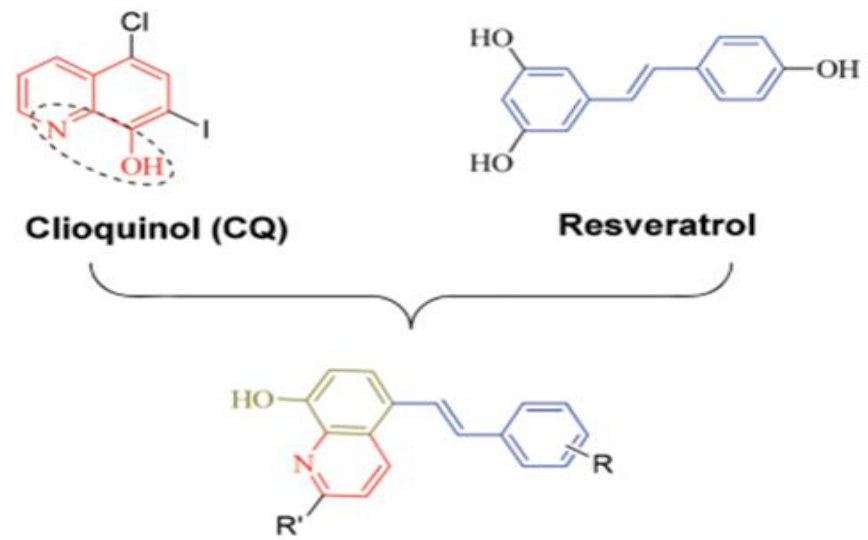

Figure 2. 


\section{Cell adhesion molecule}

Recently, genome wide association studies (GWAS) have been used to investigate AD pathogenesis. However, a large proportion of AD heritability has yet to be explained. However, it is unclear whether cell adhesion molecule (CAM) is present in the Genetic and Environmental Risk for Alzheimer's Disease Consortium (GERAD) GWAS and brain expression GWAS (Bao et al., 2015). Meanwhile, AD GWAS and AD brain expression datasets may provide complementary information to identify important pathways involved in AD (Hochstrasser et al., 2010). It also has been indicated that a systems analysis using (1) KEGG pathways, (2) large-scale AD GWAS from GERAD, (3) two brain expression GWAS datasets from the AD cerebellum and temporal cortex, and (4) previous results from pathway analysis of AD GWAS. Their results indicate that (1) CAM is a consistent signal in five AD GWAS; (2) CAM is the most significant signal in AD; (3) their confirmed previous AD risk pathways related to immune system and diseases, and cardiovascular disease, etc.; and (4) they highlighted the in $\mathrm{AD}$ for the first time. However, the genetic mechanisms of the CAM pathway in AD are unclear . In other work, Bao et al., conducted pathway analysis using (1) Kyoto Encyclopedia of Genes and Genomes and Gene Ontology pathways; (2) 4 brain expression GWAS datasets; and (3) 2 wholegenome AD case-control expression datasets. Using the 4 brain expression GWAS datasets, they identified that genes regulated by cis-regulatory single-nucleotide polymorphisms (SNPs) were significantly enriched in the CAM pathway. Interestingly, CAM is a significantly enriched pathway using down-regulated genes and all differentially expressed genes in dataset 5, and all differentially expressed genes in dataset 6. Collectively, their results show that CAM pathway genes are regulated by cisregulatory SNPs and show significantly altered expression in AD (Xiang et al., 2015).

\section{Natural products}

The complex pathology of AD includes oxidative stress, metal deposition, aggregation of amyloid and tau, and disturbances in cholinesterase (Gheysarzadeh and Yazdanparast, 2012; Gheysarzadeh and Yazdanparast, 2015). Drugs targeted toward reduction of amyloidal load have been discovered, but there is no effective pharmacological treatment for combating the disease so far. Natural products have become an important avenue for drug discovery research. Acetylcholinesterase inhibitors (AChEi) like galantamine, are the main class of drugs currently used for the treatment of AD is the only naturally occurring substance. Among this kind of drug, alkaloids, coumarins, terpenes, and polyphenols have been assessed for their anti-AChE activity(dos Santos et al., 2018).

Oxidative stress is generally accepted as one of the main paradime of AD (Gheysarzadeh and Yazdanparast, 2015). Therefor natural anti-oxidant are of the intrest for recent decades.polyphenols are natural products that have been shown to be effective in the modulation of the type of neurodegenerative changes seen in $A D$, suggesting a possible therapeutic role. For instance, scientists focuse on the chemistry of polyphenols and their role in oxidative stress and modulating amyloid precursor protein (APP) processing. They also provide new hypotheses on how these therapeutic molecules may modulate APP processing, prevent $\mathrm{A} \beta$ aggregation, and fibril destabilizing which consequently disrupt preformed fibrils (Bahramikia et al., 2012). 
Finally, the role of polyphenols in modulating Alzheimer's pathology is discussed (Caruana et al., 2016). Pharmacodynamic studies confirmed that representative compounds from these series enhance Microtubule-stabilization in the brains of wildtype mice (Figure 3). Thus, these classes of MT-stabilizers hold promise for the development of orally active, CNS-directed MT-stabilizing therapies (Lou et al., 2014b).
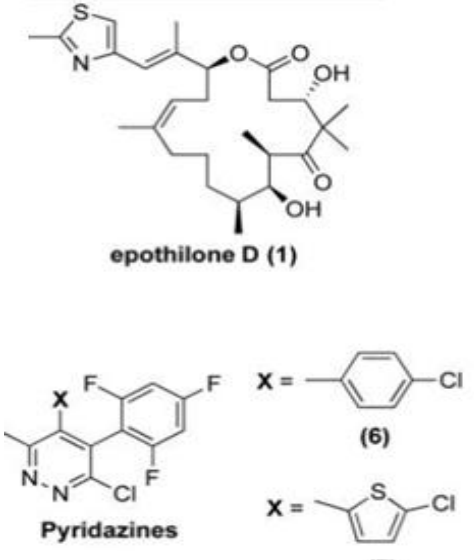

(7)

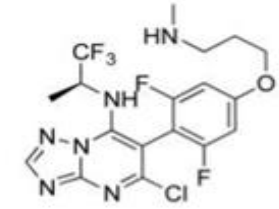

Triazolopyrimidines cevipabulin (2)<smiles>CC(C)Nc1c(-c2c(F)cc(F)cc2F)c(Cl)nc2nccnc12</smiles>
(37)<smiles>CC(Nc1nc(-c2ccccn2)nc(Cl)c1-c1c(F)cc(F)cc1F)C(F)(F)F</smiles><smiles>CC(C)c1cccc(C(C)C)c1N1C(=O)c2ccc(O)cc2C1=O</smiles>

(5)<smiles>NS(=O)(=O)c1ccc(NC(NC(=O)c2ccc(Br)cc2)C(Cl)(Cl)Cl)cc1</smiles><smiles>COc1ccccc1N1CCN(C(=O)c2cc(=O)c3cc(C)cc(C)c3o2)CC1</smiles>

Figure 3.

\section{Other AD treatment products}

Alzheimer's disease (AD) is a progressive neurodegenerative disorder. The disease leads to irreversible loss of neurons that result in reduced level of acetylcholine neurotransmitter (ACh). The reduction of ACh level impairs brain functioning. One aspect of AD therapy is to maintain ACh level up to a safe limit, by blocking acetylcholinesterase (AChE), an enzyme that is naturally responsible for its degradation. In this research, Amat-Ur-Rasool and Ahmed presents an in-silico screening and designing of hAChE inhibitors as potential anti-Alzheimer drugs. Human AChE was found to be most approptiate target site as compared to commonly used Torpedo AChE. Among the tested dietry phytochemicals, berberastine, berberine, yohimbine, sanguinarine, elemol and naringenin are the worth mentioning phytochemicals as potential anti-Alzheimer drugs. Dickkopf-related protein 1 (Dkk1), a vital antagonist of the Wnt signaling, was reported to be closely associated with bone homeostasis and osteoporosis. Interestingly, high level of Dkk1 in the brain increases the risk of AD. It is suggested that Dkk1 may be a common potent risk factor involved in osteoporosis and AD. Therefore, Guo et al., hypothesize that Dkk1 may play a role in both osteoporosis and AD. Their hypothesis will shed new light on the understanding of the relationship 
between these two diseases and help to explain some common characters of osteoporosis and AD (Guo et al., 2016).

In mid-stage male APPL/S mice, LM11A-31 administered for 3 months starting at 6-8 months of age prevented and/or reversed atrophy of basal forebrain cholinergic neurites and cortical dystrophic neurites. Importantly, a 1 month LM11A-31 treatment given to male APPL/S mice (12-13 months old) with late-stage pathology reversed the degeneration of cholinergic neurites in basal forebrain, ameliorated cortical dystrophic neurites, and normalized increased basal forebrain levels of p75NTR. Similar results were seen in female Tg2576 mice (Simmons et al., 2014).

Much effort has been devoted to developing effective therapeutic agents. Recently, targeting microRNAs (miRNAs) with small molecules has become a novel therapy for human diseases (Gheysarzadeh et al., 2018). In other research, Meng et al., present a systematic computational approach to construct a bioactive Small molecule and miRNA association Network in AD (SmiRN-AD), which is based on the gene expression signatures of bioactive small molecule perturbation and AD-related miRNA regulation. They also performed topological and functional analysis of the SmiRN-AD from multiple perspectives. At the significance level of p 0.01, 496 small molecule-miRNA associations, including $25 \mathrm{AD}$-related miRNAs and 275 small molecules, were recognized and used to construct the SmiRN-AD. The drugs that were connected with the same miRNA tended to share common drug targets and belong to the same therapeutic category. The miRNAs that were linked to the same small molecule regulated more common miRNA targets. Further analysis of the positive connections (quinostatin and miR-148b, amantadine and miR-15a) and the negative connections (melatonin and miR-30e-5p) indicated that our large-scale predictions afforded specific biological insights into AD pathogenesis and therapy. This study proposes a holistic strategy for deciphering the associations between small molecules and miRNAs in AD, which may be helpful for developing a novel effective miRNA-associated therapeutic strategy for AD (Meng et al., 2014).

Augmenting BDNF/TrkB signaling has been demonstrated to be a promising strategy for reversing cognitive deficits in preclinical models of Alzheimer disease (AD).

Analysis of GFP-labeled neurons in CaM/Tet-DTA mice revealed that 7,8-DHF induced a significant and selective increase in the density of thin spines in CA1 of lesioned mice, without affecting mushroom or stubby spines. These findings suggest chronic upregulation of TrkB signaling with 7,8-DHF may be an effective and practical strategy for improving function in $\mathrm{AD}$, even after substantial neuronal loss has occurred (Castello et al., 2014).

Metallothionein-III (MT-III) displays neuro-inhibitory activity and is involved in the repair of neuronal damage. An altered expression level of MT-III suggests that it could be a mitigating factor in Alzheimer's disease (AD) neuronal dysfunction. Currently there are limited marketed drugs available against MT-III. The inhibitors are mostly pseudopeptide based with limited ADMET. In this study, available database InterBioScreen (natural compounds) was screened out for MT-III by Roy and coworkers. In other work, the efficient high yield synthesis of novel pyridine 2,4,6-tricarbohydrazide derivatives (4a-4i) along with their a-glucosidase, acetylcholinesterase (AChE) and butyrylcholinesterase (BChE) inhibition activities were presented by Riaz research group. The enzymes inhibition results showed the potential of synthesized compounds in controlling both type-II diabetes mellitus and Alzheimer's disease. In vitro biological 
investigations revealed that most of compounds were more active against yeast aglucosidase than the reference compound acarbose. Among the tested series the compound one of the compounds bearing 4-flouro benzyl group was noted to be the most active against a-glucosidase, and it displayed weak inhibition activities against AChE and BChE. The effective Compound exhibited the most desired results against all three enzymes, as it was significantly active against all the three enzymes; a-glucosidase, AChE and BChE. Due to the most favorable activity of the effective Compound against the tested enzymes, for molecular modeling studies this compound was selected to investigate its pattern of interaction with a-glucosidase and AChE targets (Zhao et al., 2016). A more recent study used a BDNF mimetic compound called 7,8dihydroxyflavone (7,8-DHF), as a potent TrkB agonist. This compound has low bioavailability and a moderate pharmacokinetic (PK) profile (Perpetuini et al., 2019). In order to improve bioavailbility of this agents, a group of researcher used a prodrug strategy. They synthesized a large number of 7,8-DHF derivatives through ester or carbamate group modification on the catechol ring to improve effectiveness (Chen et al., 2018).

\section{Conclusions and remarks}

In the forthcoming years, it is very probable that the new compounds will be increasingly designed and synthesized to treat $\mathrm{AD}$, which is highly desirable. In this review article, initially the principles of most AD drugs design and synthesis have been described and then their applications have been reviewed.

\section{References}

Akhter H, Huang W-T, van Groen T, Kuo H-C, Miyata T, Liu R-M. 2018. A Small Molecule Inhibitor of Plasminogen Activator Inhibitor-1 Reduces Brain Amyloid- $\beta$ Load and Improves Memory in an Animal Model of Alzheimer's Disease. J Alzheimers Dis 64(2):447-457.

Alkadhi KA, Tran TT. 2015. Chronic Stress Decreases Basal Levels of Memory-Related Signaling Molecules in Area CA1 of At-Risk (Subclinical) Model of Alzheimer's Disease. Molecular Neurobiology 52(1):93-100.

Alzheimer's A. 2015. 2015 Alzheimer's disease facts and figures. Alzheimer's \& dementia: the journal of the Alzheimer's Association 11(3):332.

Angelucci F, Čechová K, Průša R, Hort J. 2019. Amyloid beta soluble forms and plasminogen activation system in Alzheimer's disease: Consequences on extracellular maturation of brain-derived neurotrophic factor and therapeutic implications. CNS Neurosci Ther 25(3):303-313.

Aziz O, Bürli RW, Fischer DF, Frearson J, Wall MD. 2015. Towards small molecules as therapies for alzheimer's disease and other neurodegenerative disorders. Drug Design and Discovery in Alzheimer's Disease. p 199-290.

Bahramikia S, Yazdanparast R, Gheysarzadeh A. 2012. Syntheses and Structure-Activity Relationships of Seven Manganese-Salen Derivatives as Anti-amyloidogenic and Fibril-destabilizing Agents Against Hen Egg-white Lysozyme Aggregation. Chem Biol Drug Des 80(2):227-236.

Bao X, Liu G, Jiang Y, Jiang Q, Liao M, Feng R, Zhang L, Ma G, Zhang S, Chen Z. 2015. Cell adhesion molecule pathway genes are regulated by cis-regulatory SNPs and show 
significantly altered expression in Alzheimer's disease brains. Neurobiol Aging 36(10):2904. e2901-2904. e2907.

Beck MW, Oh SB, Kerr RA, Lee HJ, Kim SH, Kim S, Jang M, Ruotolo BT, Lee JY, Lim MH. 2015. A rationally designed small molecule for identifying an in vivo link between metal-amyloid- $\beta$ complexes and the pathogenesis of Alzheimer's disease. Chemical Science 6(3):1879-1886.

Cao Q, Shin WS, Chan H, Vuong CK, Dubois B, Li B, Murray KA, Sawaya MR, Feigon J, Black DL. 2018. Inhibiting amyloid- $\beta$ cytotoxicity through its interaction with the cell surface receptor LilrB2 by structure-based design. Nature chemistry: 1 .

Caruana M, Cauchi R, Vassallo N. 2016. Putative role of red wine polyphenols against brain pathology in Alzheimer's and Parkinson's disease. Frontiers in nutrition 3:31.

Castello NA, Nguyen MH, Tran JD, Cheng D, Green KN, LaFerla FM. 2014. 7,8dihydroxyflavone, a small molecule TrkB agonist, improves spatial memory and increases thin spine density in a mouse model of alzheimer disease-like neuronal loss. PLoS ONE 9(3).

Chang P-T, Talekar RS, Kung F-L, Chern T-R, Huang C-W, Ye Q-q, Yang M-Y, Yu C-W, Lai S-Y, Deore RR. 2015. A newly designed molecule J2326 for Alzheimer's disease disaggregates amyloid fibrils and induces neurite outgrowth. Neuropharmacology 92:146-157.

Chen C, Wang Z, Zhang Z, Liu X, Kang SS, Zhang Y, Ye K. 2018. The prodrug of 7, 8dihydroxyflavone development and therapeutic efficacy for treating Alzheimer's disease. Proceedings of the National Academy of Sciences 115(3):578-583.

dos Santos TC, Gomes TM, Pinto BAS, Camara AL, de Andrade Paes AM. 2018. Naturally occurring acetylcholinesterase inhibitors and their potential use for Alzheimer's disease therapy. Front Pharmacol 9.

Gheysarzadeh A, Sadeghifard N, Afraidooni L, Pooyan F, Mofid MR, Valadbeigi H, Bakhtiari H, Keikhavani S. 2018. Serum-based microRNA biomarkers for major depression: MiR-16, miR-135a, and miR-1202. Journal of research in medical sciences: the official journal of Isfahan University of Medical Sciences 23.

Gheysarzadeh A, Yazdanparast R. 2012. Inhibition of H2O2-induced cell death through FOX01 modulation by EUK-172 in SK-N-MC cells. Eur J Pharmacol 697(1-3):4752.

Gheysarzadeh A, Yazdanparast R. 2015. STAT5 Reactivation by Catechin Modulates H 2 O 2-Induced Apoptosis Through miR-182/FOX01 Pathway in SK-N-MC Cells. Cell Biochem Biophys 71(2):649-656.

Guo X, Tang P, Liu P, Liu Y, Chong L, Li R. 2016. Dkk1: A promising molecule to connect Alzheimer's disease and osteoporosis. Medical Hypotheses 88:30-32.

Hochstrasser T, Weiss E, Marksteiner J, Humpel C. 2010. Soluble cell adhesion molecules in monocytes of Alzheimer's disease and mild cognitive impairment. Experimental Gerontology 45(1):70-74.

Hossain S, Hashimoto M, Katakura M, Al Mamun A, Shido 0. 2015. Medicinal value of asiaticoside for Alzheimer's disease as assessed using single-molecule-detection fluorescence correlation spectroscopy, laser-scanning microscopy, transmission electron microscopy, and in silico docking. BMC Complement Altern Med 15(1):118. 
Kumar A, Nisha CM, Silakari C, Sharma I, Anusha K, Gupta N, Nair P, Tripathi T, Kumar A. 2016. Current and novel therapeutic molecules and targets in Alzheimer's disease. J Formos Med Assoc 115(1):3-10.

Lou K, Yao Y, Hoye AT, James MJ, Cornec A-S, Hyde E, Gay B, Lee VM-Y, Trojanowski JQ, Smith III AB. 2014a. Brain-penetrant, orally bioavailable microtubule-stabilizing small molecules are potential candidate therapeutics for Alzheimer's disease and related tauopathies. J Med Chem 57(14):6116-6127.

Lou K, Yao Y, Hoye AT, James MJ, Cornec AS, Hyde E, Gay B, Lee VMY, Trojanowski JQ, Smith AB, Brunden KR, Ballatore C. 2014b. Brain-penetrant, orally bioavailable microtubule-stabilizing small molecules are potential candidate therapeutics for Alzheimer's disease and related tauopathies. Journal of Medicinal Chemistry 57(14):6116-6127.

Mao F, Yan J, Li J, Jia X, Miao H, Sun Y, Huang L, Li X. 2014a. New multi-target-directed small molecules against Alzheimer's disease: a combination of resveratrol and clioquinol. Organic \& biomolecular chemistry 12(31):5936-5944.

Mao F, Yan J, Li J, Jia X, Miao H, Sun Y, Huang L, Li X. 2014b. New multi-target-directed small molecules against Alzheimer's disease: A combination of resveratrol and clioquinol. Organic and Biomolecular Chemistry 12(31):5936-5944.

Meng F, Dai E, Yu X, Zhang Y, Chen X, Liu X, Wang S, Wang L, Jiang W. 2014. Constructing and characterizing a bioactive small molecule and microRNA association network for Alzheimer's disease. Journal of the Royal Society Interface 11(92).

Nakanishi A, Minami A, Kitagishi Y, Ogura Y, Matsuda S. 2015. BRCA1 and p53 tumor suppressor molecules in Alzheimer'S disease. International Journal of Molecular Sciences 16(2):2879-2892.

Perpetuini AC, Mathoux J, Kennedy BN. 2019. The potential of small molecule brainderived neurotrophic factor: mimetics to treat inherited retinal degeneration. Neural regeneration research 14(1):85.

Savelieff MG, Liu Y, Senthamarai RRP, Korshavn KJ, Lee HJ, Ramamoorthy A, Lim MH. 2014. A small molecule that displays marked reactivity toward copper-versus zinc-amyloid- $\beta$ implicated in Alzheimer's disease. Chemical Communications 50(40):5301-5303.

Simmons DA, Knowles JK, Belichenko NP, Banerjee G, Finkle C, Massa SM, Longo FM. 2014. A small molecule p75NTR ligand, LM11A-31, reverses cholinergic neurite dystrophy in Alzheimer's disease mouse models with mid- To late-stage disease progression. PLoS ONE 9(8).

Sun Y, Fan J, Zhu Z, Guo X, Zhou T, Duan W, Shen X. 2015. Small molecule TBTC as a new selective retinoid $\mathrm{X}$ receptor $\alpha$ agonist improves behavioral deficit in Alzheimer's disease model mice. European Journal of Pharmacology 762(1):202-213.

Wozniak MA, Frost AL, Itzhaki RF. 2013. The helicase-primase inhibitor BAY 57-1293 reduces the Alzheimer's disease-related molecules induced by herpes simplex virus type 1. Antiviral Research 99(3):401-404.

Wright JW, Kawas LH, Harding JW. 2014. The development of small molecule angiotensin IV analogs to treat Alzheimer's and Parkinson's diseases. Progress in Neurobiology. 
Wright JW, Kawas LH, Harding JW. 2015. The development of small molecule angiotensin IV analogs to treat Alzheimer's and Parkinson's diseases. Progress in Neurobiology 125:26-46.

Xiang Z, Xu M, Liao M, Jiang Y, Jiang Q, Feng R, Zhang L, Ma G, Wang G, Chen Z. 2015. Integrating genome-wide association study and brain expression data highlights cell adhesion molecules and purine metabolism in Alzheimer's disease. Mol Neurobiol 52(1):514-521.

Yu Y, Zhang L, Li C, Sun X, Tang D, Shi G. 2014a. A method for evaluating the level of soluble $\beta$-amyloid(1-40/1-42) in Alzheimer's disease based on the binding of gelsolin to $\beta$-amyloid peptides. Angewandte Chemie - International Edition 53(47):12832-12835.

Yu Y, Zhang L, Li C, Sun X, Tang D, Shi G. 2014b. A Method for Evaluating the Level of Soluble $\beta$-Amyloid (1-40/1-42) in Alzheimer's Disease Based on the Binding of Gelsolin to $\beta$-Amyloid Peptides. Angewandte Chemie International Edition 53(47):12832-12835.

Zhao H, Zhou S, Zhang M, Feng J, Wang S, Wang D, Geng Y, Wang X. 2016. An in vitro AChE inhibition assay combined with UF-HPLC-ESI-Q-TOF/MS approach for screening and characterizing of AChE inhibitors from roots of Coptis chinensis Franch. J Pharm Biomed Anal 120:235-240.

How to cite this article: Masome Rahmty, Recent Development in Synthesis and Pharmacological Evaluation of Small Molecule to Treat Alzheimer's Diseases: A Review Study. International Journal of Advanced Biological and Biomedical Research, 2020, 8(2), 134145. Link: http://www.ijabbr.com/article 36889.html 